\title{
A FRAMEWORK FOR TIME-DRIVEN ACTIVITY-BASED COSTING FOR ORTHOPEDIC PROCEDURE
}

\author{
Bayan Holozadah', Shaligram Pokharel $^{2}$ \\ ${ }^{1}$ Graduate Student, Engineering Management Program, Qatar University, Qatar \\ 2Professor, Department of Mechanical and Industrial Engineering, Qatar University, Qatar \\ (bh1206761@qu.edu.qa,shaligram@qu.edu.qa)
}

\section{Abstract}

The study used method of Time-Driven Activity-Based costing (TDABC) approach to examined cost reduction in healthcare sector, Knee Arthroplasty Department in Al-Emadi Hospital as case-study for delivering a primary knee arthroplasty consultation. The purpose of the study is to propose an optimization model that tends to determine significant and insignificant resources and cost uncertainties in a particular healthcare service. Then compare results of optimization model with current costing methods used in healthcare. Eight-steps of TDABC method are applied to obtain Capacity Cost Rate (CCR) of each human resource involved in care-delivery cycle. Data was collected from interviewing staff and patients, financial reports, and human resource reports. Multiple linear regression (MLR) model is used to test strength of relationship between time and cost variable. Optimization model applied to decrease uncertainties by using Least Square method. Optimized model showed that human resources are not fully utilized which leads to error in costing model. Seven human resources out of eight are significant to model. Accuracy of optimized model is equal to $3 \%$, with RMSE equal to 6 . Total cost of the optimized model equal $\$ 177,492.45$ which is better simulate actual cost $\$ 180,048$. The research value is about building a new statistical model using MLR analysis to predict behavior and data trend of main healthcare segmentation: Human Resource. Also, propose a generic optimization model that can be used for specific healthcare service costing.

Keywords: Traditional Accounting; ABC; TDABC; Healthcare Accounting

\section{Introduction}

- Kaplan and Porter developed an 8-steps framework for costing method called Time Driven Activity-Based Costing (TDABC) which used to present the true cost and enhancing costing efficiency in healthcare.

- TDABC uses time as a main cost driver and follows the assumption that most resources have their own capacities, which can be identified in term of time (Namazi, 2009).

- TDABC fall to have some inaccuracies in cost model due to studying the entire system, while method can be augmented by explicit consideration of the details of costs of human resources.

- TDABC method assume that all human resources have $80 \%$ utilization rate, which leads to inaccuracies in cost model as staff are being either underutilized or overutilized.

- Optimized model needs to be built in order to test the strength of relationship between the time and cost variables the human resources consume with patients and see how total cost will be affected by knowing which human resource is significantly affecting the total cost and which does not have high impact on the total cost.

\section{Methods}

- Data used in this study are collected from interviewing staff and patients, financial reports, and human resource reports

The study used quantitative design model to understand relationship between time and cost of human resources and how they could affect total cost of healthcare

The study used random sampling for survey of 1000 patients who visited knee Arthroplasty for pre-operation office and pre-operation testing and consulting during the fiscal year 2019.

Lucidchart application was used to draw the detailed process map of the patient care flow which indicates activities, resources encounter during care-delivery cycle, and time used by each patient during each activity.

TDABC method 8-steps framework are applied to identify the CCR and the total cost.

MLR model applied to test and assess the strength of relationship between cost and time human resources consume with patients to see which human resource are directly affecting the total cost of healthcare.

Sensitivity analysis done to examine the utilization of human resources with an increase of utilization by $5 \%$, starting from $70 \%$ up to $90 \%$, and working hours from 6 hours until 10 hours working by 1 hour increase.

Actual cost and predicted cost examined to minimize the error between them. Then a new CCR of the optimization model are introduced and compared with TDABC CCR and regression model CCR.

\section{Results}

Descriptive analysis as shown in Figure 1 reveled that there is a huge number of minutes are wasted but paid without actual work, and in this time many other patients could be served.

Figure 2 shows that there is a gap between TDABC cost and actual cost. TDABC cost is overestimated as it compared to the actual cost as well as TDABC does not simulate the actual cost data.

Correlation and directionality tested with each resource and found that all the resources have highly positive correlation $(0.63)$ between the two identified variables unless security resource.

- The MLR model equation that used to calculate the total cost is built by using CCR as coefficient as shown in Equation 1.

$R^{2}$ for the given data when applying MLR is equal to 0.3971 , which indicate that the actual cost the hospital obtain are not closed to the predicted cost after the regression analysis done.

- Sensitivity analysis shows that as the utilization of resource increase, the CCR will decrease, and consequently the total cost will decrease.

- Accuracy (MAPE) of the optimized model is equal to 3\%, and RMSE value was equal to 6 .

- The optimized model is shown in Equation 2.

The total cost of each model is presented in Table 1.

The CCR of each model is presented in Table 2 .

Resources that were having a low utilization become lower more. However, the resources that were having a high utilization, now they are within the acceptable utilization rate of resources.

$$
\text { Table } 1 \text { Total Cost }
$$

\begin{tabular}{|c|c|c|c|c|}
\hline & $\begin{array}{c}\text { TDABC } \\
\text { Model }\end{array}$ & $\begin{array}{c}\text { Regression } \\
\text { Model }\end{array}$ & $\begin{array}{c}\text { Optimized } \\
\text { Model }\end{array}$ & $\begin{array}{c}\text { Actual } \\
\text { Cost }\end{array}$ \\
\hline Total cost $\mathbf{( \$ )}$ & $150,168.3$ & $166,044.84$ & $177,492.45$ & 180,048 \\
\hline
\end{tabular}
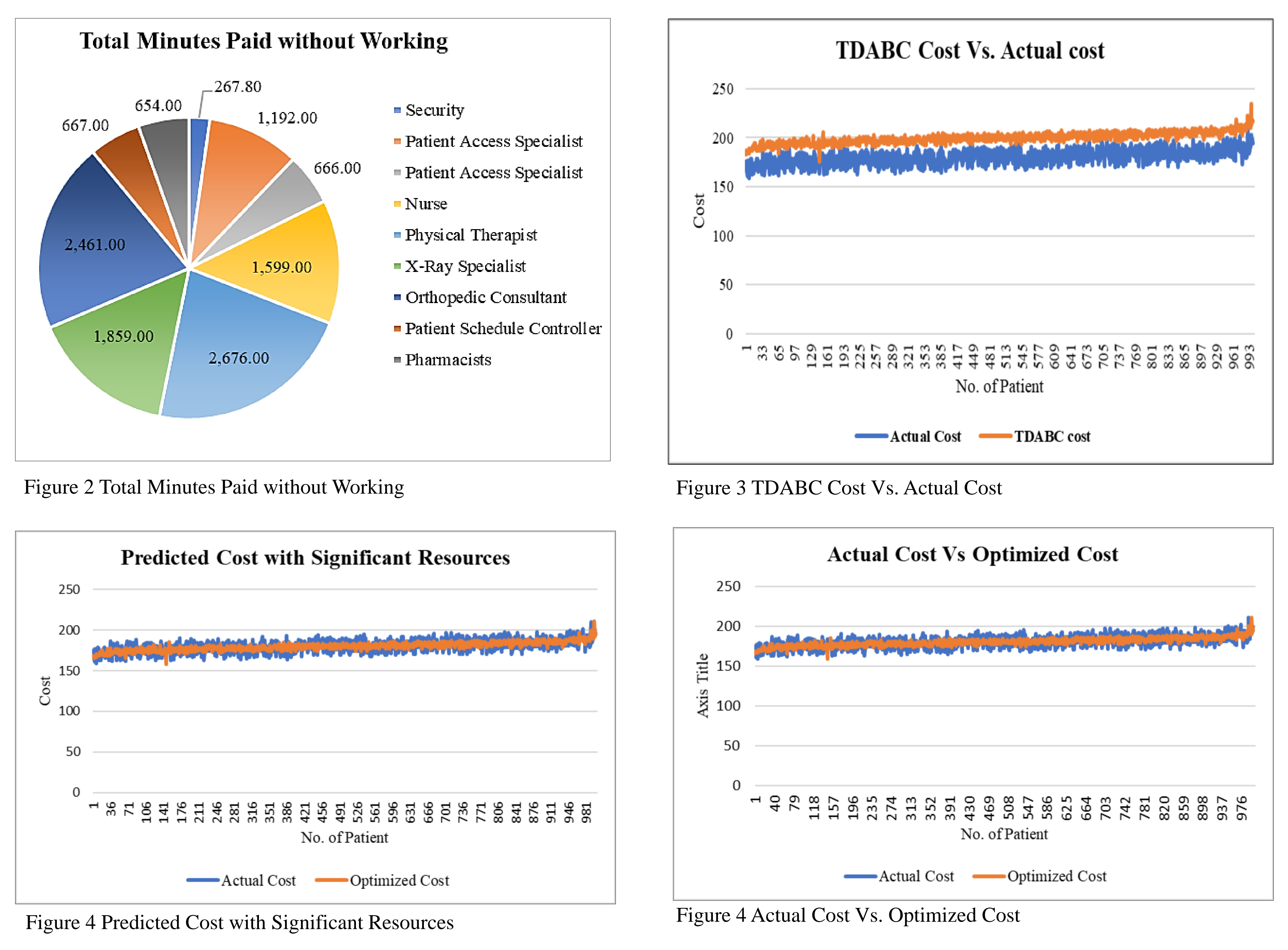

Table 2 CCR of each Model

\begin{tabular}{llll} 
'Resource & TDABC CCR & Regression Model CCR & Optimized CCR \\
\hline Security & 0.08 & 0.16 & 0.16 \\
Patient special & 0.32 & 0.44 & 0.48 \\
Nurse & 0.54 & 0.47 & 0.53 \\
Physical Therapist & 0.53 & 0.67 & 0.7 \\
X-Ray Specialist & 0.45 & 0.59 & 0.65 \\
Orthopedic Consultant & 1.81 & 1.98 & 2.06 \\
Patient Schedule Coordinator & 0.38 & 0.52 & 0.59 \\
Pharmacist & 0.9 & 0.68 & 0.84 \\
\hline
\end{tabular}

\section{Conclusion}

The research tested 8 hypothesis that were built on two main research questions which are: Does the cost of human resource significantly affect the total cost of the Knee Arthroplasty department?, and What methods are available to resource cost based on care delivery time handling with TDABC method?.

- For the 7 human resources integrated in the patient care-delivery cycle, it is verified that the time consumed by them can significantly influence the total cost.

- The validation of the model was done by taking another sample of 200 patients that are following a caredelivery in the knee-arthroplasty department. The model was validated by two concepts which are MAPE and RMSE. It founds that MAPE $=7.1 \%$, and RMSE $=15.56$.

The time human resource spends with the patient while delivering the care consider as a main factor to understand the utilization of each resource which if optimized will reveal a more accurate total cost and cost reduction.

\section{Future work}

. Adopting the model developed in a large patient population would be good in benchmarking to have a more accurate factors and thus more accurate total cost.

2. Using the cost-model to better enhance the system in different way such as value-based model and ensure quality of care.

3. Apply lean method to the Knee Arthroplasty department on patient flow to determine whether this new cost model affect the quality and flow of patient or not.

\section{Acknowledgement}

This poster was possible because of the god's grace, and the follow-up done by my Supervisor Dr. Shaligram Pokharel. I would like to express my special thanks of gratitude to my family who give support to me along the journey. 